UCRL--97451

DEBB 013098

\title{
SYNTHESIS OF ACCRETION DISK AND \\ NONTHERMAL SOURCE MODELS FOR AGN
}

\author{
DAVID L. BAND \\ IGPP-LLNL, L-419, Livermore, CA 94550 \\ AND MATTHEW A. MALKAN \\ UCLA, Department of Astronomy, Los Angeles, CA 90024
}

\section{DISCLAIMER}

This report was prepared as an account of work sponsored by an agency of the United States Government. Neitber the United States Government nor any agency thereof, nor any of their employees, makes any warranty, express or implied, or assumes any legal liability or responsibility for the accuracy, completeness, or usefulness of any information, apparatus, product, or process disclosed, or ropresents that its use would not infringe privately owned rights. Referenee berein to any specific commercial product, process, or service by trade name, trademark, manufacturer, or otherwise does not necessarily constitute or imply its endorsement, recommendation, or favoring by the United States Government or any agency thereof. The views and opinions of authors expressed herein do not necessarily state or reflect those of the United States Government or any agency thereol.

To be published in

Active Galactic Nuclei:

Proceedings of the Georgia State University Conference

eds. H. R. Miller and P. J. Wiita, (Berlin: Springer-Verlag)

November, 1987 


\title{
SYNTHESIS OF ACCRETION DISK AND NONTHERMAL SOURCE MODELS FOR AGN
}

\author{
DaVid L. Band ${ }^{1}$ and MatThew A. MaLKaN ${ }^{2}$ \\ ${ }^{1}$ IGPP-LLNL, L-419, Livermore, CA 94550 \\ ${ }^{2} U C L A$, Department of Astronomy, Los Angeles, CA 90024
}

A scenario for the central engine of AGN has been developed consisting of a massive black hole $(\mathrm{MBH})$ onto which gas accretes through an accretion disk. The accretion disk radiates the observed optical and ultraviolet continua. Surrounding the MBH is a nonthermal source which produces the infrared and soft $\mathrm{X}$-ray continua by synchrotron emission, and the $\mathrm{X}$-ray spectrum by inverse Compton scattering of the optical-ultraviolet photons from the accretion disk. Previously we modeled the accretion disk (M.A.M.) and nonthermal source (D.L.B.) separately, and here we combine the two models to form a unified description of the AGN engine. This combined model can be inverted to determine source parameters from observed spectra. A group of AGN for which multiband observations exist car then be modeled to: demonstrate the validity of the combinad model for a large number of objects; establish the range of parameter values that describe the source; and search for any correlations between source description and type.

An accretion disk around a MBH is the most promising origin of the optical-ultraviolet "big blue bump." The methodology has been developed by Sun and Malkan (1987a,b) and is described by Sun in the present proceedings. The spectrum is modeled by assuming that the accretion disk is geometrically thin and optically thick. At each radius either a black body or a low surface-gravity non-LTE stellar atmosphere radiating the necessary energy flux is used to calculate the spectrum from that radius. The stellar atmospheres are less efficient radiators than a black body, and consequently they emit at a higher frequency than would a black body. Gravitational defocusing (light that passes close to the black hole is bent) and Doppler beaming (the light from the rapidly rotating material at the center of the disk is beamed along the disk) is included, providing a strong inclination dependence. In particular, observers near the plane of the disk see a significantly harder spectrum than those who view the disk face on. Both stationary Schwartzschild and maximally rotating Kerr black holes can be modeled.

The nonthermal model is a variant of the Synchrotron-Self Compton (SSC) model and differs from it in two respects: a) the soft photons which are scattered into the $\mathrm{X}$-ray band originate in the "big blue bump" and not the synchrotron source; and b) the electron distribution is assumed to consist of low energy flat and high energy steep components (Band and Grindlay 1985, 1986 and Band 1987). The nonthermal source is modeled as a sphere of radius $R$ surrounding the accretion disk (for the sake of simplicity the accretion disk is assumed to be a point source at the center). The source is permeated by a tangled magnetic field $B$ (allowing the field to be treated as a scalar). Within the source is a homogeneous "broken" electron density: $n_{e}=n \gamma^{-p}, 1 \leq \gamma \leq \gamma_{b}$ and $n_{a}=n \gamma_{b} \gamma^{-p-1}, \gamma_{b} \leq \gamma_{b}$, where $\gamma=E / m c^{2}$. This electron distribution could arise due to injection $\propto \gamma^{-p}$, with radiative losses above $\gamma_{b}$ steepening the distribution. The resulting spectrum is: 
- Synchrotron self-absorbed below $\nu_{T}$

- A flat synchrotron power law between $\nu_{T}$ and $\nu_{b}$ with $L_{\nu} \propto \nu^{-\alpha}$ where $\alpha=(p-1) / 2$.

- A steep synchrotron power law above $\nu_{b}$ with $L_{\nu} \propto \nu^{-a-1 / 2}$.

- A flat X-ray power law due to inverse Compton scattering by the flat component of the electron distribution. The flat scattered component extends to $\gamma_{b}^{2} \nu_{c}$, where $\nu_{c}$ is the characteristic frequency of the soft photon source.

- The gamma ray spectrum is affected by the break in the electron distribution, the decrease in the Klein-Nishina cross section, and absorption due to pair creation.

The equations for the various spectral features can be inverted to derive the source parameters from the observed spectrum. The observed spectrum from the far-infrared through X-rays is fit with the following components (see example of such a fit in the Figure below):

- Balmer jump and blended Fe II lines due to emission in the Broad Line Region

- Flux normalization of starlight from host galaxy

- Absorption in both the Milky Way and the host galaxy

- Accretion disk defined by: $M_{B}$-black hole mass; $\dot{m}$-accretion rate; and $\mu_{i}$-cosine of disk inclination angle to the line of sight.

- Synchrotron spectrum defined by: $\alpha$-spectral index of steep component; $L_{\nu}\left(\nu_{f i d}\right)$-normalization of steep component at fiducial $\nu_{\text {fidi }} \nu_{b}$-break frequency between that and steep components; and $\nu_{T}$-turnover frequency due to synchrotron sialf-absorption.

This spectral decomposition gives the parameters for the accretion disk model, e.g., $M_{B}$ and $\dot{m}$. The synchrotron turnover frequency $\nu_{T}$, break frequency $\nu_{b}$, and normalization $L_{\nu}\left(\nu_{f i d}\right)$ are used in conjunction with an $\mathrm{X}$-ray flux $L_{x}$ and a moment of the accretion disk luminosity to derive the parameters of the non-thermal source: the normalization of the electron distribution $n$, the magnetic field $B$, and the source radius $R$.

Preliminary fits to five objects are given in the Table below. In all cases the accretion disk was assumed to be face on. The sources show remarkably similar parameters: $n \sim 10^{7} \mathrm{~cm}^{3}, B \sim 1000$ gauss, $R \sim 2 \times 10^{15} \mathrm{~cm}$ or about 30 Schwarzschild radii, black hole masses of a few times $10^{8} M_{\odot}$, and total luminosities of a large fraction of the Eddington limit. The break energy $\gamma_{b} \sim 50-90$ is perhaps an indication of the microphysics within the source. Finally, the magnetic and photon fields are in approximate equipartition, while the relativistic electron energy density is much smaller. The compactness $l=\left(L_{a 00} / R\right)\left(\sigma_{T} / m c^{3}\right)$ is quite large. However, $L_{a 00}$ is the ultraviolet accretion disk luminosity, whereas it is the usually unobserved luminosity at $\nu \sim m c^{2} / h$ that is relevant to the production of electron-positron pairs (Band 1987).

This work is supported by the U.S. Department of Energy, under contract No. W-7405-ENG48 to the Lawrence Livermore National Laboratory (D.L.B) and the NSF, under grant AST-8614510 (M.A.M.).

\section{References}

Band, D. L., and Grindlay, J. E. 1985, Ap. J., 298, 128.

$$
\text { 1986, Ap. J., 308, } 576 .
$$

Band, D. L. 1987, Ap. J., 321, 80. 
sun, W.H., and Malkan, M.A. 1987a, in Supermassive Black Eoles, M. Kafatos, ed. (Cambridge: Cambridge University Press). 1987b, in Active Galactic Nuclei, Neutron Stars, and Jets: Astrophysics of the Central Engines, W. Kundt, ed. (Dordrecht: Reidel), p. 124.

\section{Table-Preliminary Fits}

\begin{tabular}{|c|c|c|c|}
\hline Mkn 841 & II $Z_{W} 136$ & 3C249.1 & Mkn 1383 \\
\hline
\end{tabular}

Fitted Parameters

\begin{tabular}{|c|c|c|c|c|c|}
\hline$n_{6}=n / 10^{6} \mathrm{~cm}^{-3}$ & 19.2 & 12.4 & 7.2 & 19.3 & 9.4 \\
\hline$B_{2}=B / 100$ gauss & 13.3 & 17.0 & 8.9 & 8.2 & 12.0 \\
\hline$R_{15}=R / 10^{15} \mathrm{~cm}$ & 0.8 & 1.2 & 1.9 & 1.1 & 5.2 \\
\hline $\boldsymbol{\gamma}_{b}$ & 56. & 53. & 83. & 89. & 82. \\
\hline$M_{\mathrm{B}}=M_{B} / 10^{8} M_{\odot}$ & 0.7 & 1.1 & 8.0 & 1.6 & 5.0 \\
\hline$\dot{m}=\dot{M} /\left(M_{\odot-y r^{-1}}\right)$ & 0.15 & 0.41 & 1.0 & 0.56 & 14.0 \\
\hline Underlying $\alpha$ & 0.73 & 0.80 & 0.62 & 0.65 & 0.85 \\
\hline$L_{\text {ace }}$ ergs-sec -1 & $1.9 \times 10^{45}$ & $5.4 \times 10^{45}$ & $1.4 \times 10^{46}$ & $7.5 \times 10^{45}$ & $7.8 \times 10^{46}$ \\
\hline \multicolumn{6}{|l|}{ Derived Quantities } \\
\hline$u_{a} \operatorname{ergs}-\sec ^{-1}$ & 10.8 & 6.3 & 4.7 & 12.1 & 4.5 \\
\hline$u_{B}$ ergs-sec -1 & $7.0 \times 10^{4}$ & $1.2 \times 10^{5}$ & $3.2 \times 10^{4}$ & $2.7 \times 10^{4}$ & $5.7 \times 10^{4}$ \\
\hline urv ergs-sec ${ }^{-1}$ & $3.8 \times 10^{3}$ & $3.0 \times 10^{4}$ & $3.1 \times 10^{4}$ & $4.9 \times 10^{4}$ & $2.3 \times 10^{4}$ \\
\hline$R / R_{S c h}$ & 37. & 37. & 8.1 & 23. & 35. \\
\hline$L_{\text {ado }} / L_{\text {Edd }}$ & 0.27 & 0.49 & 0.18 & 0.47 & 1.56 \\
\hline$l=\left(L_{\mathrm{aco}} / R\right)\left(\sigma_{\mathrm{T}} / \mathrm{mc}^{3}\right)$ & 68. & 122. & 200. & 185. & 406. \\
\hline
\end{tabular}

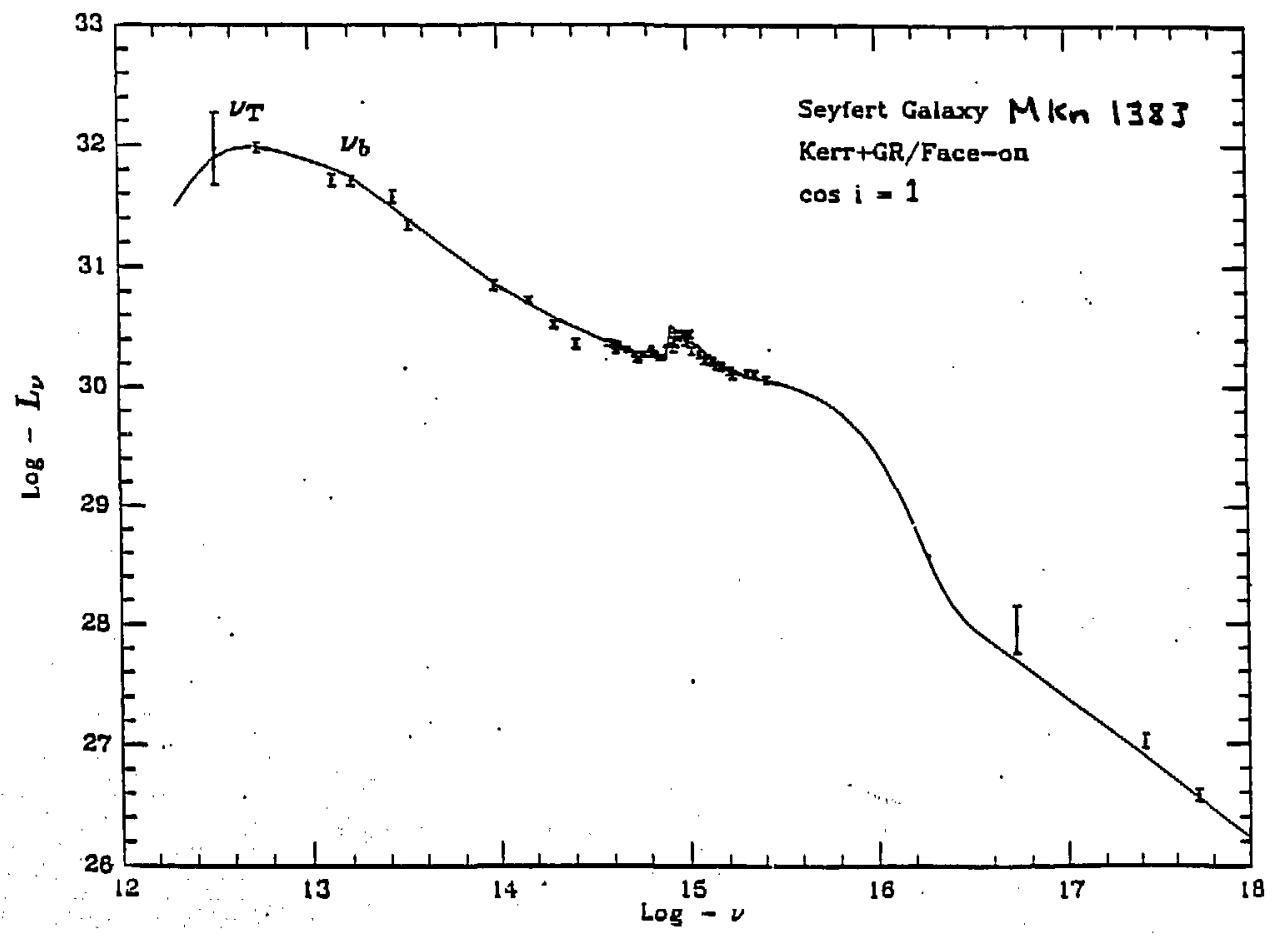

Figure: Fit to observed spectrum of the Seyfert Mkn 1383.' 